\title{
Biomarkers of brain ischemia-reperfusion injury after carotid endarterectomy
}

\author{
Marek Iłżecki', Marcin Feldo', Anna Bogucka-Kocka², Daniel Zalewski ${ }^{2}$, Paulina Chmiel $^{2}$, \\ Shawn Dave ${ }^{3}$, Joanna Iłżecka ${ }^{4}$, Tomasz Zubilewicz' \\ 'Chair and Department of Vascular Surgery and Angiology, Medical University of Lublin, Poland \\ ${ }^{2}$ Chair and Department of Biology and Genetics, Medical University of Lublin, Poland \\ ${ }^{3}$ University of Oklahoma Health Sciences Center in Oklahoma City, Oklahoma, USA \\ ${ }^{4}$ Department of Neurology, Neurological and Psychiatric Nursing, Medical University of Lublin, Poland
}

\begin{abstract}
Introduction: Endarterectomy of the internal carotid artery (CEA) is a surgical procedure used to prevent cerebral ischemic stroke. Available data from previous literature indicates that CEA may lead to complications in the form of cerebral ischemia-reperfusion syndrome and oxidative stress. The aim of this study was to evaluate the serum levels of oxidative stress-related biomarkers such as 8-hydroxydeoxyguanosine (8-OHdG) and malondialdehyde (MDA) in patients who underwent CEA.
\end{abstract}

Material and methods: Twenty-four patients with severe internal carotid artery stenosis participated in the study. Serum samples were taken from patients at three different times: within 24 hours preoperatively to CEA, 12 hours postoperatively, and 48 hours postoperatively. Serum 8-OHdG and MDA levels were measured using a commercially available enzyme-linked immunosorbent assay.

Results: Serum 8-OHdG levels indicated statistically significant elevation 12 hours after surgery when compared to preoperative levels $(p<0.05)$. A further increase in the concentration of this parameter was observed 48 hours after surgery when compared to the previous measurement, however, this increase was no longer statistically significant $(p=0.05)$. Furthermore, serum MDA levels also indicated a statistically significant elevation 48 hours after surgery when compared to preoperative levels $(p<0.05)$.

Conclusion: Our study showed that CEA causes an increase in blood levels of 8-OHdG and MDA, which may be related to the occurrence of oxidative stress during cerebral ischemia-reperfusion injury. Therefore, 8-OHdG and MDA may represent corresponding markers of cerebral ischemia-reperfusion complications in patients undergoing CEA.

Key words: brain ischemia-reperfusion injury, carotid endarterectomy, 8-hydroxydeoxyguanosine, malondialdehyde, oxidative stress biomarkers

Acta Angiol 2021; 27, 4: 113-119

\section{Introduction}

Acute ischemic stroke is a leading cause of morbidity and mortality worldwide, and the incidence of ischemic stroke is predicted to increase in the coming years. The carotid atherosclerotic occlusive disease accounts for up to $20 \%$ of all ischemic strokes, and mounting evidence suggests that, in the setting of an acute ischemic stroke due to carotid disease, earlier treatment with carotid intervention results in better outcomes [I].

Address for correspondence: Marek Iłżecki, MD, PhD, Chair and Department of Vascular Surgery and Angiology, Medical University of Lublin, Staszica St. I , 20-08I Lublin, Poland, e-mail: marekilzecki@umlub.pl 
Carotid endarterectomy (CEA) is the procedure of choice for reducing the risk of ischemic stroke in both symptomatic and asymptomatic carotid artery stenosis. However, subclinical cerebral microemboli can occur during these procedures and perioperative stroke is a known complication of CEA [2-5]. Cerebral hyperperfusion syndrome is a rare but significant complication after carotid revascularization and is an increasingly recognized downstream consequence following acute stroke treatments. Although the pathophysiology of hyperperfusion syndrome is incompletely understood, current theories suggest cellular damage occurs due to an increase in cerebral blood flow and impaired cerebral autoregulation, particularly in the areas of a disrupted blood-brain barrier, in addition to baroreceptor dysfunction during carotid surgery [6]. Postoperative vasculopathy, including reversible cerebral vasoconstriction syndrome, is a rare complication of carotid intervention and may be an under-identified cause of neurologic deficit after revascularization [7]. Intracerebral hemorrhage in the context of cerebral hyperperfusion syndrome is an uncommon but potentially lethal complication after carotid revascularization for carotid occlusive disease $[8,9]$. Ischemic stroke and ischemia-reperfusion injury are associated with multiple insults to the cerebral microcirculation, including reactive oxygen species overproduction, leukocyte adhesion and infiltration, brain blood barrier disruption, and capillary hypoperfusion; ultimately resulting in tissue edema, hemorrhage, brain injury, and delayed neuron damage [10].

Oxidative stress is one of the mechanisms involved in ischemic brain injury induced by ischemia-reperfusion. In addition to the direct cytotoxic effects of lipid peroxidation, oxidative DNA damage occurs immediately after ischemia-reperfusion and leads to ischemic brain injury, as reflected by neuronal death and brain edema. Increased levels of 8-hydroxy-2'-deoxyguanosine (8-OHdG), classically a repair product in the oxidation of guanine in DNA, indicates direct oxidative damage of DNA. The oxidatively modified $8-\mathrm{OHdG}$ product is considered one of the predominant forms of free radical-induced lesions of DNA [1 I-15]. Oxidative DNA damage has been proposed to be a causative factor in blood-brain barrier dysfunction and is known to induce neuronal degeneration and apoptosis $[16,17] .8-\mathrm{OHdG}$ can be detected in human tissue, blood samples, or urine and is considered a biomarker of generalized and cellular oxidative stress [1I, 18]. Previous studies have reported that peripheral concentrations of $8-\mathrm{OHdG}$ are a valuable indicator of the severity of oxidative brain damage in acute cerebral infarction [19].

Malondialdehyde (MDA) is produced from polyunsaturated fatty acids (PUFAs) both by chemical reactions and by reactions catalyzed by enzymes. Therefore,
MDA has been suggested to be the prototype of the so-called thiobarbituric acid reactive substances (TBARS). MDA is an end-product formed during lipid peroxidation due to degradation of cellular membrane phospholipids. During oxidative stress, MDA is released into the extracellular space and resultantly into the blood. Therefore, MDA has been used as an effective biomarker of lipid oxidation and subsequently an important indicator of oxidative stress. In many disease states, higher concentrations of MDA are measured in biological samples when compared to healthy, nonpathological samples. Higher circulating levels of MDA have been previously noted in patients with history of ischemic stroke when compared to their controlled counterparts. Additionally, elevated serum MDA levels in patients with malignant middle cerebral artery infarction were found to be directly associated with early mortality in those respective patients [20, 2I].

The objective of our study was to report the changes of oxidative stress-related biomarkers such as 8-OHdG and MDA in the serum of patients undergoing CEA.

\section{Material and methods}

The study was conducted with the approval of the Ethical Committee of the Medical University of Lublin (KE-0254/218/2014). All patients gave informed consent to participate in the study.

The study included patients of the Department of Vascular Surgery and Angiology who underwent surgery for internal carotid artery stenosis. Carotid artery Doppler studies were performed in all patients and met qualifications for surgical intervention according to the European Society of Vascular Surgery guidelines using the NASCET (North American Symptomatic Carotid Endarterectomy Trial) criteria [22, 23].

Twenty-four patients, aged $5 \mathrm{I}$ to 88 years with a mean age of 69 years, were included in the study. Each patient underwent a neurological examination before and after surgery, which showed no clinical sequelae of the surgical procedure. The degree of internal carotid artery stenosis in the patients ranged from $75 \%$ to $85 \%$. Historical data indicated 6 patients with a history of ischemic stroke and 4 patients with a history of transient ischemic attack. In the remaining patients, carotid artery stenosis was asymptomatic. Patients with organic brain damage in the course of other nervous system diseases were excluded from the study. For statistical calculations, men ( 16 patients) and women (8 patients) were separated and patients were divided according to age; either younger ( $\leq 69$ years; 13 patients) or older (> 69 years; II patients).

Blood samples were collected from the antecubital vein of patients at three different times: within 24 hours 


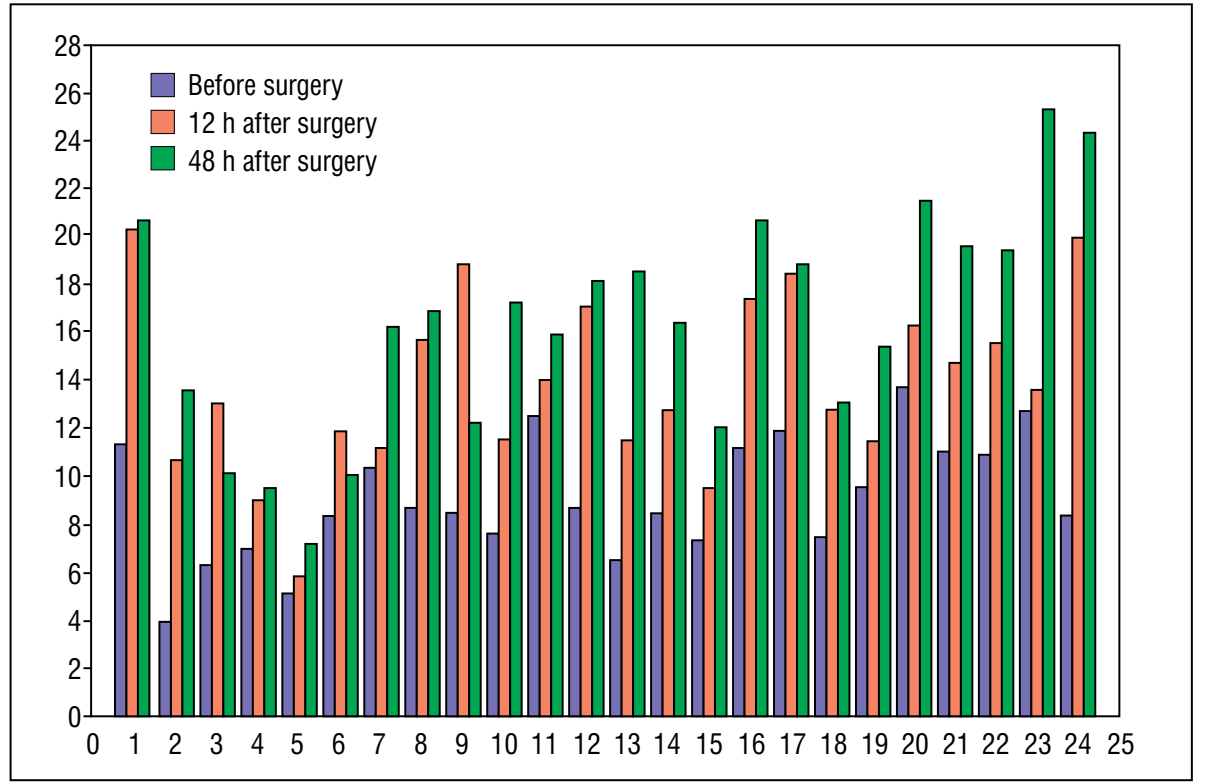

Figure I. Serum 8-OHdG $[\mathrm{ng} / \mathrm{ml}]$ in patients

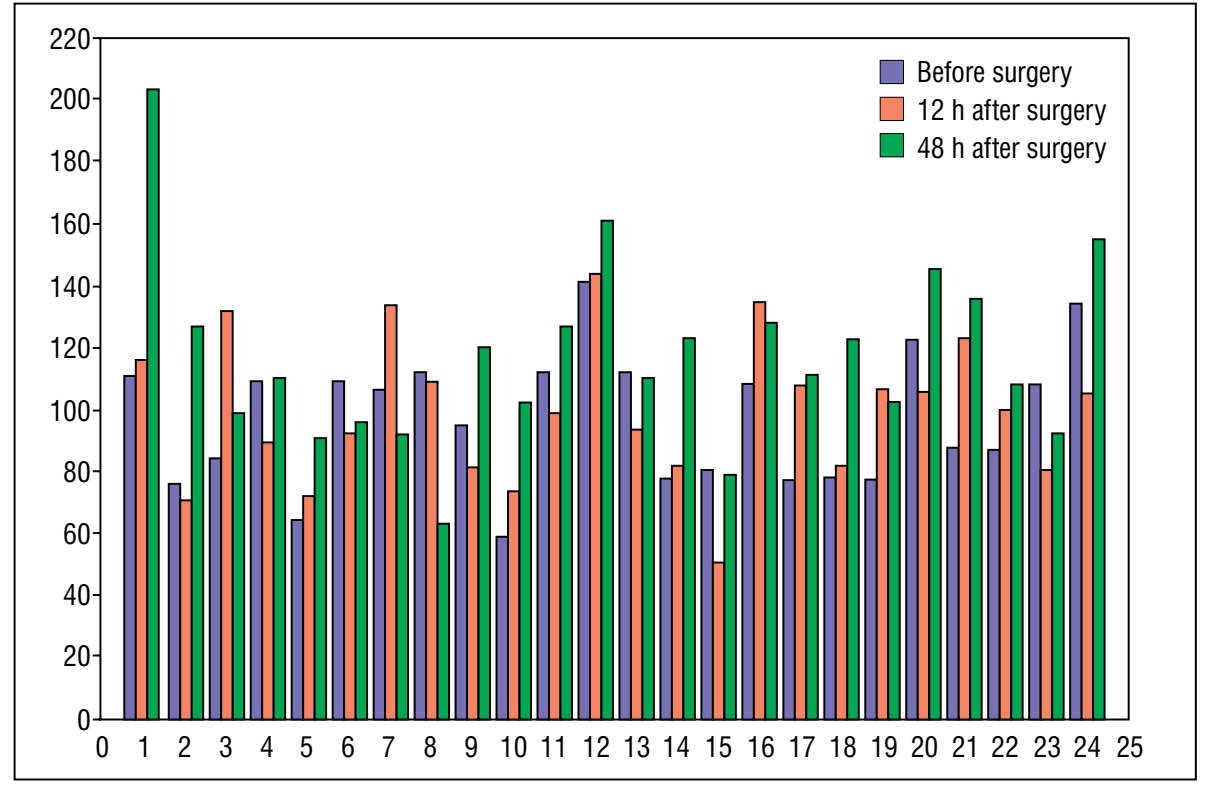

Figure I. Serum MDA $[\mathrm{ng} / \mathrm{ml}]$ in patients

before CEA surgery $[A], 12$ hours after surgery $[B]$, and 48 hours after surgery $[C]$. Serum $8-O H d G$ and MDA levels were measured using a commercially available immunoassay 8-OHdG (8-Hydroxydeoxyguanosine) ELISA Kit, and Human MDA (Malonyldehyde) ELISA Kit; (Wuhan Fine Biotech Co., Ltd., China).

Statistical analysis was performed using STATISTICA software, version 12 (StatSoft, Inc., Poland). The distribution of the collected data was evaluated using the Shapiro-Wilk test. 8-OHdG and MDA levels data were analyzed using ANOVA with Tuckey HSD post hoc test. For dichotomous system, Student's t-test was used to evaluate the differences between groups. 8-OHdG and MDA levels were expressed in $\mathrm{ng} / \mathrm{ml}$. Results were deemed statistically significant if $p<0.05$.

\section{Results}

Serum 8-OHdG and MDA levels in patients are presented in Figures I, 2. 
Table I. Serum 8-OHdG and MDA levels in patients, and a comparative analysis

\begin{tabular}{|c|c|c|c|c|}
\hline \multirow[t]{2}{*}{ Biomarker } & \multicolumn{3}{|c|}{ Mean and SD [ng/ml] } & \multirow[t]{2}{*}{ Comparison } \\
\hline & Before surgery [A] & I2 $\mathrm{h}$ after CEA [B] & $48 \mathrm{~h}$ after CEA [C] & \\
\hline 8-OHdG & 9.03 SD 2.50 & 13.83 SD 3.67 & I6.35 SD 4.68 & $\begin{array}{c}{[A-B-C] p=0.0000 *} \\
{[A-B] p=0.0001 *} \\
{[B-C] p=0.05} \\
{[A-C] p=0.0001 *}\end{array}$ \\
\hline MDA & 97.05 SD 21.44 & 99.13 SD 23.52 & I I 6.86 SD 29.38 & $\begin{aligned} {[A-B-C] p } & =0.013^{*} \\
{[A-B] p } & =0.95 \\
{[B-C] p } & =0.04^{*} \\
{[A-C] p } & =0.02^{*}\end{aligned}$ \\
\hline
\end{tabular}

ANOVA and post hoc Tuckey HSD test; *statistically significant

The ANOVA test revealed that the sampling time significantly affects serum $8-O H d G$ levels $(p<0.05)$. Serum 8-OHdG levels were statistically and significantly elevated 12 hours after surgery when compared to preoperative levels $(p<0.05)$. A further increase in the level of this parameter was observed 48 hours after surgery when compared to the previous measurement. However, this latter increase was no longer statistically significant $(p=0.05)$. Additionally, the ANOVA test also indicated that the sampling time significantly affects serum MDA levels ( $p<0.05)$. Serum MDA levels were statistically and significantly elevated 48 hours after surgery compared to 12 hours after surgery, and when compared to preoperative levels $(p<0.05)$ (Table I).

There was no statistically significant difference in serum $8-\mathrm{OHdG}$ and MDA levels between male and female groups or between elderly and younger patients $(p>0.05)$.

\section{Discussion}

Oxidative stress during CEA is one of the proposed mechanisms that results in changes in cerebral perfusion and likely contributes to acute brain ischemia (ABI). The increased production of reactive oxygen species and reactive nitrogen species during $A B I$ may cause an unregulated inflammatory response, and further lead to structural and functional injury of neurons [24].

Our study showed that elevations in serum 8-OHdG levels were statistically significant 12 hours after surgery when compared to preoperative levels. A further increase in the level of this parameter was observed 48 hours after surgery compared to the previous measurement. However, this latter increase was no longer statistically significant.

Data from previous literature on 8-OHdG suggests its function as a marker of oxidative stress after ischemia-reperfusion brain injury. Liu et al. [19] assessed the correlation between plasma $8-O H d G$, as a marker of oxidative DNA damage, and progressive brain damage in rats subjected to transient or permanent ischemia. Plasma 8-OHdG significantly increased at I $2 \mathrm{~h}$ and peaked at $24 \mathrm{~h}$ after reperfusion. This increase was directly proportional to increased infarct volume, DNA degradation, and reflected immunohistochemical findings in the cortical region. In the permanent middle cerebral artery occlusion (MCAO) model, plasma 8-OHdG levels were associated with the brain contents of 8-OHdG. Plasma $8-\mathrm{OHdG}$ and the cortical infarct volume were lower in the 0.5 - and I-h than the 2-h MCAO model. The authors suggest that 8-OHdG may function as a peripheral biomarker and may be an indicator of oxidative brain damage in acute cerebral infarction.

According to Lorente et al. [25], secondary injury due to oxidation may occur during ischemic stroke; possibly leading to oxidative damage of deoxyribonucleic acid (DNA) and ribonucleic acid (RNA). Higher blood concentrations of 8-OHdG (through the oxidation of guanosine from DNA) have been found in ischemic stroke patients than in healthy subjects, as well as in patients with and without post-ischemic stroke depression.

In the study conducted by Liu et al. [26], 24I ischemic stroke patients were consecutively recruited within the first $24 \mathrm{~h}$ of stroke symptom onset and were followed up at I month. Serum 8-OHdG and catalase (CAT) levels were measured within $24 \mathrm{~h}$ of hospital admission. Serum levels of $8-\mathrm{OHdG}$ and CAT were noted to be increased in depressed patients at the time of admission. A positive correlation was found between the 8-OHdG and CAT levels when compared to the total number of stroke patients, respectively.

Nakajima et al. [14] evaluated the utility of urinary 8-OHdG, an oxidative stress marker, to demonstrate 
an association between the changes of 8-OHdG and outcomes after acute ischemic stroke. In the overall cohort, the mean urinary level of $8-\mathrm{OHdG}$ on day 7 was increased than that on day 0 . The $8-\mathrm{OHdG}$ levels on day 0 were no different between those patients who experienced poor outcomes when compared to those with good outcomes. However, a notable increase in the 8-OHdG levels from day 0 to 7 in stroke patients with poor outcomes was significantly higher when compared to those with good outcomes. The authors concluded that the biochemical concentration changes of $8-\mathrm{OHdG}$ may be related to oxidative stress and may be considered a marker of ischemic brain injury which may furthermore reflect the clinical prognosis of ischemic stroke.

The results of the study conducted by Zhao et al. [27] demonstrated that obvious neuron damage was accompanied by the expression of autophagic markers LC3 and Beclin-I, and that the formation of 8-OHdG and autophagosomes were significantly increased in the brain cortex after ischemia-reperfusion.

Our study showed that elevations of serum MDA levels were statistically significant $\mathbf{4 8}$ hours after surgery when compared to preoperative levels.

Suga et al. [28] showed that hyperperfusion was observed immediately after CEA in 13\% of patients. Reduced preoperative cerebrovascular reactivity (CVR) and an increase in MDA-LDL after internal carotid artery (ICA) declamping were significantly associated with the development of postoperative cerebral hyperperfusion among the variables tested. Ten of I I patients with reduced preoperative CVR and increased MDALDL after ICA declamping developed post-CEA hyperperfusion, and 2 of these patients developed cerebral hyperperfusion syndrome. The authors concluded that both preoperative cerebral hemodynamic impairment and reactive oxygen species produced during surgery directly correlate with the development of cerebral hyperperfusion after CEA.

The purpose of the study conducted by Saito et al. [29] was to determine whether or not concentrations of MDA-modified low-density lipoprotein (LDL), a biochemical marker of oxidative damage, in the jugular bulb during CEA correlates with the development of postoperative cognitive impairment in patients undergoing CEA. The MDA-LDL concentrations at 5 and 20 minutes after ICA declamping were both significantly higher than concentrations before ICA clamping. The authors concluded that increased concentrations of MDA-LDL in the jugular bulb during CEA directly correlated with development of postoperative cognitive impairment.

In the study conducted by Shaafi et al. [30] serum MDA concentrations were found to be significantly higher in the ischemic stroke group when compared to the control group. High levels of MDA were associated with increased development of stroke; however, increased levels of MDA were not associated with having risk factors for stroke. Additionally, MDA levels were correlated with the modified Rankin Scale score at follow-up.

The aim of the study conducted by Cojocaru et al. [3 I] was to evaluate oxidative stress in patients with acute ischemic stroke. The authors observed significantly higher values of MDA in the first 24 hours, as well as significantly higher values of MDA at 7 days.

In the next study, fifty patients with a history of acute ischemic stroke were compared with a control group in a case-control study. The results indicated significantly increased serum MDA levels in those with a history of acute ischemic stroke. The authors concluded that oxidative stress plays a major role in the etiopathogenesis of acute ischemic stroke, and the deranged oxidant-antioxidant balance further contributes to disease severity [32].

In the study conducted by et al. [33], MDA levels were increased in acute ischemic stroke patients within $24 \mathrm{~h}$ after stroke onset versus control groups. Additionally, MDA levels were also significantly higher in ischemic stroke patients than in control groups in other studies [34, 35].

There are studies in the literature that have demonstrated the efficacy of antioxidant treatment after ischemia-reperfusion brain injury, providing evidence that oxidative stress plays an important role in disease prognosis. The study conducted by Zhou et al. [36] indicated that Schizandrin-A protects against cerebral ischemia-reperfusion injury by suppressing inflammation and oxidative stress and that this effect is regulated by the AMPK/Nrf2 pathway. In another study, protocatechualdehyde was found to protect against cerebral ischemia-reperfusion-induced oxidative injury, and that this neuroprotective effect involves the $\mathrm{PKC} \varepsilon /$ /Nrf2/HO-I pathway [37]. Suda et al. [38] revealed that valproic acid attenuates ischemia-reperfusion injury in the rat brain through inhibition of oxidative stress and inflammation. Moreover, Nagatani et al. [39] investigated the effect of hydrogen gas $(\mathrm{H} 2)$, a strong free radical scavenger, on the survival rate of mice following global cerebral ischemia-reperfusion. Nagatani et al. observed that $\mathrm{H} 2$ treatment significantly improved the 7-day survival rate of mice. Furthermore, the beneficial effects of $\mathrm{H} 2$ treatment on brain injury were associated with significantly lower levels of oxidative stress markers (8-OHdG and MDA) in the brain tissue.

Kalimeris et al. [40] examined the antioxidant role of propofol in ischemia-reperfusion during CEA and its influence on cognitive dysfunction after CEA. The 
authors concluded that propofol seemed to improve cognitive performance after CEA. This improvement was associated with decreased indices of ischemic cerebral damage and seemed to be due to the antioxidative effect in the ischemic cerebral circulation.

In conclusion, our study showed a significant increase in serum 8-OHdG and MDA levels in patients following CEA. The observed increase in these parameters may represent a response to the onset of oxidative stress as a result of cerebral ischemia-reperfusion injury. Thus, $8-\mathrm{OHdG}$ and MDA may be indicative markers of cerebral ischemia-reperfusion injury after CEA. In light of the data presented above, it is conceivable that the use of neuroprotective antioxidants after surgery may prove to be beneficial in patients undergoing CEA.

\section{Conflict of interest}

None.

\section{References:}

I. Brinster CJ, Sternbergh WC. Safety of urgent carotid endarterectomy following thrombolysis. J Cardiovasc Surg (Torino). 2020; 6I (2): 149-158, doi: 10.23736/5002 I-9509.20. I I 179-0, indexed in Pubmed: $32225 \mid 34$.

2. Khattar NK, Friedlander RM, Chaer RA, et al. Perioperative stroke after carotid endarterectomy: etiology and implications. Acta Neurochir (Wien). 2016; 158(12): 2377-2383, doi: 10.1007/s00701-016-2966-2, indexed in Pubmed: 2769600I.

3. Reddy RP, Karnati T, Massa RE, et al. Association between perioperative stroke and 30-day mortality in carotid endarterectomy: A meta-analysis. Clin Neurol Neurosurg. 2019; 181: 44-51, doi: 10.1016/j.clineuro.2019.03.028, indexed in Pubmed: 30986726.

4. Hitchner E, Baughman BD, Soman S, et al. Microembolization is associated with transient cognitive decline in patients undergoing carotid interventions. J Vasc Surg. 2016; 64(6): 1719-1725, doi: 10.1016/j.jvs.2016.06.104, indexed in Pubmed: 27633169.

5. Zhou W, Baughman BD, Soman S, et al. Volume of subclinical embolic infarct correlates to long-term cognitive changes after carotid revascularization. J Vasc Surg. 2017; 65(3): 686-694, doi: 10.1016/j.jvs.2016.09.057, indexed in Pubmed: 28024850.

6. Kirchoff-Torres KF, Bakradze E. Cerebral Hyperperfusion Syndrome After Carotid Revascularization and Acute Ischemic Stroke. Curr Pain Headache Rep. 2018; 22(4): 24, doi: 10. I007/ s। I916-018-0678-4, indexed in Pubmed: 29556806.

7. Shoskes A, Mullaguri N, Purohit M, et al. Ipsilateral Reversible Cerebral Vasculopathy Secondary to Carotid Artery Revascularization: A Case Report. Neurohospitalist. 2021; II(2): 165-169, doi: 10.1177/194/874420966850, indexed in Pubmed: 33791063.

8. Galyfos G, Sianou A, Filis K. Cerebral hyperperfusion syndrome and intracranial hemorrhage after carotid endarterectomy or carotid stenting: A meta-analysis. J Neurol Sci. 2017; 38I: 74-82, doi: 10.1016/j.jns.2017.08.020, indexed in Pubmed: 28991720.
9. Abreu P, Nogueira J, Rodrigues FB, et al. Intracerebral hemorrhage as a manifestation of cerebral hyperperfusion syndrome after carotid revascularization: systematic review and meta-analysis. Acta Neurochir (Wien). 2017; 159(II): 2089-2097, doi: 10.1007/s00701-017-3328-4, indexed in Pubmed: 28916863.

10. Sun K, Fan J, Han J. Ameliorating effects of traditional Chinese medicine preparation, Chinese materia medica and active compounds on ischemia/reperfusion-induced cerebral microcirculatory disturbances and neuron damage. Acta Pharm Sin B. 2015; 5(I): 8-24, doi: 10.1016/j.apsb.2014.11.002, indexed in Pubmed: 26579420.

II. Yeh TT, Hsieh YW, Wu CY, et al. The reliability and predictive ability of a biomarker of oxidative DNA damage on functional outcomes after stroke rehabilitation. Int J Mol Sci. 2014; 15(4): 6504-65।6, doi: 10.3390/ijms |5046504, indexed in Pubmed: 24743892.

12. Vendrov $A E$, Vendrov $K C$, Smith $A$, et al. Oxidative stress and vascular disease. Arterioscler Thromb Vasc Biol. 2005; 25(I): 29-38, doi: 10.1161/01.ATV.0000150649.39934.13, indexed in Pubmed: 15539615.

13. Chamorro A. Role of inflammation in stroke and atherothrombosis. Cerebrovasc Dis. 2004; 17 Suppl 3: I-5, doi: 10.1 I59/000075297, indexed in Pubmed: 1473025 I.

14. Nakajima $\mathrm{H}$, Unoda $\mathrm{KI}$, Ito $\mathrm{T}$, et al. The Relation of Urinary 8-OHdG, A Marker of Oxidative Stress to DNA, and Clinical Outcomes for Ischemic Stroke. Open Neurol J. 2012; 6: 5I-57, doi: $10.2|74 /| 874205 \times 0|2060| 005 \mid$, indexed in Pubmed: 22754596.

15. Canugovi C, Misiak M, Ferrarelli LK, et al. The role of DNA repair in brain related disease pathology. DNA Repair (Amst). 2013; 12(8): 578-587, doi: 10.1016/j.dnarep.2013.04.010, indexed in Pubmed: 23721970.

16. Takemori K, Murakami T, Kometani T, et al. Possible involvement of oxidative stress as a causative factor in blood-brain barrier dysfunction in stroke-prone spontaneously hypertensive rats. Microvasc Res. 2013; 90: 169-172, doi: 10.1016/j. mvr.2013.08.005, indexed in Pubmed: 23978333.

17. Radi E, Formichi P, Battisti C, et al. Apoptosis and oxidative stress in neurodegenerative diseases. J Alzheimers Dis. 2014; 42 Suppl 3: S125-SI52, doi: 10.3233/JAD-132738, indexed in Pubmed: 25056458.

18. Xiang F, Shuanglun $X$, Jingfeng $W$, et al. Association of serum 8-hydroxy-2'-deoxyguanosine levels with the presence and severity of coronary artery disease. Coron Artery Dis. 201 I; 22(4): 223-227, doi: 10.1097/MCA.0b013e328344b6/5, indexed in Pubmed: 21407076.

19. Liu H, Uno M, Kitazato KT, et al. Peripheral oxidative biomarkers constitute a valuable indicator of the severity of oxidative brain damage in acute cerebral infarction. Brain Res. 2004; 1025(I-2): 43-50, doi: 10.1016/j.brainres.2004.07.07I, indexed in Pubmed: 15464743.

20. Tsikas D. Assessment of lipid peroxidation by measuring malondialdehyde (MDA) and relatives in biological samples: Analytical and biological challenges. Anal Biochem. 2017; 524: 13-30, doi: 10.1016/j.ab.2016.10.021, indexed in Pubmed: 27789233.

21. Lorente L, Martín MM, Abreu-González P, et al. Serum malondialdehyde levels in patients with malignant middle cerebral artery infarction are associated with mortality. PLoS One. 2015 
I0(5): e0 I25893-3277, doi: 10.137//journal.pone.0125893, indexed in Pubmed: 25933254.

22. Liapis CD, Bell PRF, Mikhailidis D, et al. ESVS Guidelines Collaborators. ESVS guidelines. Invasive treatment for carotid stenosis: indications, techniques. Eur J Vasc Endovasc Surg. 2009; 37(4 Suppl): I-19, doi: 10.1016/j.ejvs.2008.11.006, indexed in Pubmed: 19286127.

23. Staikov IN, Arnold M, Mattle HP, et al. Comparison of the ECST, CC, and NASCET grading methods and ultrasound for assessing carotid stenosis. European Carotid Surgery Trial. North American Symptomatic Carotid Endarterectomy Trial. J Neurol. 2000; 247(9): 68I-686, doi: 10.1007/s004I50070I I0, indexed in Pubmed: 11081806.

24. Obradovic M, Zafirovic S, Essack M, et al. Antioxidant enzymes expression in lymphocytes of patients undergoing carotid endarterectomy. Med Hypotheses. 2020; 134: 109419, doi: 10. 1016/j. mehy.2019.109419, indexed in Pubmed: 31622925.

25. Lorente L, Martín MM, González-Rivero AF, et al. DNA and RNA oxidative damage are associated to mortality in patients with cerebral infarction. Med Intensiva (Engl Ed). 202I; 45(I): 35-4I, doi: 10.1016/j.medin.2019.07.008, indexed in Pubmed: 31492477.

26. Liu Z, Cai Y, He J. High serum levels of 8-OHdG are an independent predictor of post-stroke depression in Chinese stroke survivors. Neuropsychiatr Dis Treat. 2018; 14: 587-596, doi: I0.2I47/NDT.SI55I44, indexed in Pubmed: 29497302.

27. Zhao $Y$, Huang $G$, Chen $S$, et al. Folic acid deficiency increases brain cell injury via autophagy enhancement after focal cerebral ischemia. J Nutr Biochem. 2016; 38: 41-49, doi: 10.1016/j. jnutbio.2016.08.009, indexed in Pubmed: 27721।I5.

28. Suga $Y$, Ogasawara $K$, Saito $H$, et al. Preoperative cerebral hemodynamic impairment and reactive oxygen species produced during carotid endarterectomy correlate with development of postoperative cerebral hyperperfusion. Stroke. 2007; 38(10): 27|2-27| 7, doi: 10.1 161/STROKEAHA. 107.483495, indexed in Pubmed: I7761927.

29. Saito H, Ogasawara K, Komoribayashi N, et al. Concentration of malondialdehyde-modified low-density lipoprotein in the jugular bulb during carotid endarterectomy correlates with development of postoperative cognitive impairment. Neurosurgery. 2007; 60(6): 1067-73; discussion 1073, doi: 10.1227/0I. neu.0000277। 78.288 I3.d3, indexed in Pubmed: 1753838I.

30. Shaafi S, Hadisi F, Mahmoudinezhad M, et al. The significance of the oxidative stress markers in the one-year prognosis of patients with acute ischemic stroke: a case-control study. BMC Neurol. 202I; 2 I(I): 258, doi: 10.1 I86/s | 2883-02I-02257-x, indexed in Pubmed: 34215195.
31. Cojocaru IM, Cojocaru M, Sapira V, et al. Evaluation of oxidative stress in patients with acute ischemic stroke. Rom J Intern Med. 20I3; 5 I (2): 97-106, indexed in Pubmed: 242948I3.

32. Jena I, Nayak SR, Behera S, et al. Evaluation of ischemiamodified albumin, oxidative stress, and antioxidant status in acute ischemic stroke patients. J Nat Sci Biol Med. 2017; 8(I): II0-II3, doi: 10.4I03/0976-9668.198346, indexed in Pubmed: 28250685.

33. Milanlioglu A, Aslan M, Ozkol H, et al. Serum antioxidant enzymes activities and oxidative stress levels in patients with acute ischemic stroke: influence on neurological status and outcome. Wien Klin Wochenschr. 2016; 128(5-6): 169-174, doi: 10.1007/ s00508-015-0742-6, indexed in Pubmed: 25854910.

34. Menon B, Ramalingam K, Kumar R. Evaluating the Role of Oxidative Stress in Acute Ischemic Stroke. J Neurosci Rural Pract. 2020; II (I): 156-159, doi: 10.1055/s-0039-3402675, indexed in Pubmed: 32140020.

35. Kotur-Stevuljevic J, Bogavac-Stanojevic N, Jelic-Ivanovic Z, et al. Oxidative stress and paraoxonase $I$ status in acute ischemic stroke patients. Atherosclerosis. 2015; 24I(1): 192-198, doi: 10.1016/j.atherosclerosis.2015.05.016, indexed in Pubmed: 2601645I.

36. Zhou F, Wang M, Ju J, et al. Schizandrin A protects against cerebral ischemia-reperfusion injury by suppressing inflammation and oxidative stress and regulating the AMPK/Nrf2 pathway regulation. Am J Transl Res. 2019; II(1): 199-209, indexed in Pubmed: 30787979

37. Guo C, Wang S, Duan J, et al. Protocatechualdehyde Protects Against Cerebral Ischemia-Reperfusion-Induced Oxidative Injury Via Protein Kinase $\mathrm{C} \varepsilon / \mathrm{Nrf2} / \mathrm{HO}-\mathrm{I}$ Pathway. Mol Neurobiol. 2017; 54(2): 833-845, doi: 10.1007/s I2035-016-9690-z, indexed in Pubmed: 26780453.

38. Suda S, Katsura Ki, Kanamaru T, et al. Valproic acid attenuates ischemia-reperfusion injury in the rat brain through inhibition of oxidative stress and inflammation. Eur J Pharmacol. 2013; 707(I-3): 26-31, doi: 10.1016/j.ejphar.2013.03.020, indexed in Pubmed: 23541723.

39. Nagatani K, Wada K, Takeuchi S, et al. Effect of hydrogen gas on the survival rate of mice following global cerebral ischemia. Shock. 2012; 37(6): 645-652, doi: 10.1097/SHK. Ob0I3e3I824ed57c, indexed in Pubmed: 22392। 46.

40. Kalimeris K, Kouni S, Kostopanagiotou G, et al. Cognitive function and oxidative stress after carotid endarterectomy: comparison of propofol to sevoflurane anesthesia. J Cardiothorac Vasc Anesth. 2013; 27(6): 1246-1252, doi: 10.1053/j. jvca.20 12.12.009, indexed in Pubmed: 23725684. 